\title{
Maternal metabolic risk factors and their association with birthweight and cord blood insulin
}

\author{
Jingya Wang ${ }^{1}$, Yashu Kuang ${ }^{2}$, Malcolm Price ${ }^{3}$, Jinhua $\mathrm{Lu}^{2}$, Naveed Sattar ${ }^{4}$, Jianrong $\mathrm{He}^{2}$, \\ Marta Pittavino ${ }^{5}$, Songying Shen ${ }^{2}$, Huimin Xia ${ }^{2}$, Xiu Qiu ${ }^{2}$, KK Cheng ${ }^{3}$, and Krishnarajah \\ Nirantharakumar ${ }^{6}$ \\ ${ }^{1}$ Affiliation not available \\ ${ }^{2}$ Guangzhou Women and Children's Medical Center \\ ${ }^{3}$ University of Birmingham \\ ${ }^{4}$ University of Glasgow \\ ${ }^{5}$ University of Geneva \\ ${ }^{6}$ University of Birmingham Edgbaston Campus
}

May 5, 2020

\begin{abstract}
Objective To quantify the inter-dependency between maternal metabolic risk factors and their association with birthweight and cord blood insulin (CBI) level. Design Prospective cohort study. Setting Guangzhou Women and Children's Medical Centre (GWCMC). Population Pregnant women with a singleton pregnancy who delivered at GWCMC between Jan 2015 and Jun 2016 and had umbilical cord blood retained (total 1522). Methods Multivariable linear regression and Additive Bayesian Network analysis were used to investigate the association between maternal metabolic risk factors (pre-pregnancy body mass index [BMI], fasting glucose, lipid profiles, and early gestational weight gain [GWG]) and their interdependency in predicting birthweight and CBI concentrations. Main outcome measures Birthweight and cord blood insulin. Results High maternal pre-pregnancy BMI was strongly associated with neonatal birthweight (standardized adjusted regression coefficient $[\beta$ std] $=0.27,95 \% \mathrm{CI} 0.22-0.32$ ) directly; and with CBI indirectly. Maternal fasting glucose was positively associated with increased CBI $(\beta \mathrm{std}=0.12,95 \% \mathrm{CI}$ 0.07-0.17). Maternal GWG was positively associated with increased birthweight, but not with CBI. None of the maternal lipids profile was independently associated with birthweight or CBI. Conclusions Maternal pre-pregnancy overweight/obesity is the most influential upstream metabolic risk factor for both maternal and neonatal metabolic health, therefore weight management should be addressed from the preconception period. Maternal dyslipidaemia appears to be secondary to maternal metabolic dysfunction with no clear causality relationship with metabolic adverse outcomes in neonates.
\end{abstract}

\section{Tweetable abstract}

The most efficient way to optimize maternal and neonatal metabolic health is to address weight management before pregnancy.

\section{Introduction}

Maternal metabolic risk factors, including high pre-pregnancy body-mass-index(BMI), excessive gestational weight gain(GWG), and gestational hyperglycaemia, are known to induce adverse pregnancy outcomes. 
These include stillbirth, pre-term delivery, low or high birthweight, pre-eclampsia, and maternal postnatal diabetes/cardiovascular disease $(1,2)$. The prevalence of gestational diabetes mellitus(GDM) varies from 2 to 25 percent worldwide, and has increased over the last decades in parallel with the increasing obesity prevalence of women in child-bearing age $(3,4)$.

A variety of strategies targeting metabolic management during pregnancy have been developed to prevent endocrine-related adverse pregnancy outcomes. However, a recent individual patient data meta-analysis suggested that diet and lifestyle interventions in pregnancy only achieved modest success in reducing GWG, but have no effects on composite maternal and foetal outcomes, such as pre-term birth, macrosomia, and foetal adiposity(5). It might be partly attributed to the alterations of maternal/placental function and metabolic programming in preconception and early pregnancy, which occurs prior to when most interventions are initiated. Other than maternal weight and glucose level, maternal lipid levels during pregnancy are recognised as ignored risk factors for adverse pregnancy outcomes recently(6).

The mechanism of how maternal metabolic dysfunction is linked with neonatal health remains uncertain, however, most researchers believe it may be explained by the unfavourable intrauterine nutritional environment $(7,8)$. Previous studies mainly focused on one specific metabolic trait or on the number of metabolic disorders, without assessing the underlying interacted effect of the natural metabolic network $(9,10)$. This is due to the limited analytical ability of classical statistical methods to analyse multidimensional data. Understanding how the metabolic network influences neonatal health is crucial to future interventional studies and potentially to antenatal/prenatal advice.

In this study, we investigated the independent association of maternal metabolic traits with birthweight and cord blood insulin in the Born in Guangzhou Cohort Study(BIGCS). The inter-dependency of maternal metabolic risk factors and their association with birthweight and cord blood insulin was then mapped by Additive Bayesian Network(ABN) analysis, a robust data-driven structure discovery model, which has been widely used in other disciplines $(11,12)$.

\section{Materials and Methods}

\section{Participants}

The design and methods of BIGCS have been described previously(13). In brief, eligible women with Chinese nationality, living in Guangzhou who are $<20$ weeks gestation and who intend to deliver at one of the two Guangzhou Women and Children's Medical Centre(GWCMC) campuses were recruited into BIGCS. This study was conducted in a planned subgroup of BIGCS in whom maternal and cord blood were analysed for metabolic parameters separately. Pregnant women attending BIGCS with a singleton pregnancy who delivered at GWCMC between Jan 2015 and Jun 2016 and had umbilical cord blood retained are eligible for this study. Women were excluded if: 1) maternal blood samples unavailable at 14-27 gestation week; 2) no records of maternal fasting glucose at 20-28 gestation week; 3) lacking maternal demographic information; 4) diagnosed with health condition prior to pregnancy, including type 1 or type 2 diabetes, thyroid dysfunction, hypertension, virus hepatitis, and renal diseases. The study was powered for the association between maternal triglycerides (the potential weakest risk factors among maternal metabolic traits) with birthweight according to literature(Supplementary file S1). The eligible mother-child pairs were then selected into this study by computer generated randomization. Ethical permission for the study was granted by the GWCMC Ethics Committee.

\section{Study Procedures}

Maternal demographic data, including anthropometric measures, socioeconomic status, family and personal medical history, were collected through a semi-structured questionnaire(Q1) at recruitment. Maternal 
overnight fasting blood samples were collected during second trimester. At 22-28 weeks gestation, women attending their second prenatal visit underwent a standard $2 \mathrm{~h} 75 \mathrm{~g}$ oral glucose tolerance test(OGTT). Women with OGTT results which met or exceeded at least one threshold of the International Association of Diabetes and Pregnancy Study Groups(IADPSG) criteria(FPG[?]5.1 mmol/L, $1 \mathrm{~h}$ glucose [?]10.0 mmol/l, and $2 \mathrm{~h}$ glucose[?] $8.5 \mathrm{mmol} / \mathrm{L}$ ) were diagnosed as having gestational diabetes mellitus(GDM)(14). For participating children, birth information, including birth characteristics, delivery mode, and perinatal outcomes were obtained from routine medical records. Umbilical cord blood samples were collected by midwives at birth.

\section{Demographic Data}

Maternal demographic information(age, height, pre-pregnancy weight, parity, date of last menstrual period, monthly income, education levels, and ethnicity) were collected through Q1 questionnaire. BMI was calculated by dividing weight in kilograms by height in meters squared. Based on the recommendations of the China Obesity Task Force of the Chinese Ministry of Health, maternal pre-pregnancy BMI is classified into two groups: lean group $\left(<24 \mathrm{~kg} / \mathrm{m}^{2}\right)$ and overweight $\operatorname{group}\left([?] 24 \mathrm{~kg} / \mathrm{m}^{2}\right)(15)$. Maternal second trimester weight was measured to the nearest $0.1 \mathrm{~kg}$ using an electronic scale. Maternal early gestational weight gain(GWG) was calculated by subtracting pre-pregnancy weight from maternal second trimester weight, with documentation of the gestational age at measurement. Maternal fasting glucose concentration was obtained from OGTT test zero-time value in hospital records.

\section{Biochemical Test}

Sample collection, delivery, pre-treatment, and measurements were blinded. All blood samples were stored and delivered to pre-treatment laboratory centre. Blood samples were then separated to serum and plasma by immediate centrifugation, and were stored in EDTA tube in the bio-bank at -80 until analysis. Plasma lipids(TC, HDL-C, LDL-C, and TG) and insulin levels were measured using commercial kits in fully automated clinical analyser(Roche Diagnostics, Mannheim, Germany). Intra- and inter-day coefficients of variation(CVs) were consistently less than 2 percent for all assays.

\section{Neonatal anthropometry}

Gestational age was estimated from ultrasound examination during the first- or second-trimester. Birthweight and other information, including gestational age at delivery, mode of delivery, neonatal sex, and pregnancy complications were obtained from hospital records. Birthweight was measured to the nearest 50g using an electronic scale by midwives immediately after delivery. Birthweight Z-Score and percentile(adjusted for gestational age at delivery and neonatal sex) were calculated using Intergrowth $21^{\text {st }}$ Newborn Size Standard and Tools(16). Large for gestational age(LGA) was defined as a birthweight larger than the $90^{\text {th }}$ percentile for gestational age by sex, while Small for gestational age(SGA) was defined as a birthweight smaller than the $10^{\text {th }}$ percentile based on the same birthweight reference.

\section{Statistical Analysis}

\section{Classic statistical methods}

For the baseline table data are summarized as mean \pm Standard Deviation(SD), median(Inter Quartile Range, IQR), or counts with percentages. Pearson correlation was used to assess the impact of the long-term $-80{ }^{\circ} \mathrm{C}$ storage on insulin concentrations in EDTA tube. Adjustments were then made to account for any degradation by correcting the initial value using linear regression methods(Supplementary file S2). Similarly, maternal lipid levels were adjusted for gestational age using regression model to account for timing of blood sampling(Supplementary file S3)(17). 
Initially, linear and logistic regression were used to estimate the association between maternal metabolic parameters and neonatal continuous and binary outcomes, respectively. Further analyses using linear regression model were performed after all exposures were transformed to Z-Scores. This was to enable comparison of the effect size each maternal metabolic parameter had on birthweight Z-Score and CBI Z-Score. CBI and maternal triglycerides were log-transformed prior to standardization. Multiple imputation was used to handle missing data. Subgroup analyses were conducted in boys and girls respectively. Sensitivity analyses were conducted to compare the estimate differences between GDM and non-GDM participants, fasting blood samples and non-fasting samples, primiparous women and non-primiparous women, lean and overweight group, as well as before and after multiple imputation(Supplementary file S4). All statistical tests were two-tailed and a $\mathrm{P}<0.05$ was considered statistically significant. Statistical analyses were performed in Stata version 14.0(College Station, Texas, USA).

\section{Additive Bayesian Networks (ABN) analysis}

To further assess the inter-dependency between maternal metabolic risk factors and their association with birthweight and CBI, Additive Bayesian Network(ABN) model - an unsupervised machine learning method was conducted. Bayesian network analysis is a form of structure discovery statistical modelling that derives, from empirical data, a graphical network describing the dependency structure between variables, shown as directed acyclic graphs(DAGs)(12). ABNs comprise of DAGs where each node in the graph comprises a generalized linear model(GLM) or a generalized linear mixed model(GLMM). ABN model is suitable for analysing highly complex epidemiological data comprising many inter-dependent variables(11).

Ten variables were chosen for ABN based on prior knowledge gained from literature and findings of the classical statistical analyses. These ten variables were maternal age, maternal pre-pregnancy BMI, maternal fasting glycaemia in OGTT, early GWG, maternal fasting plasma HDL-C and triglycerides in the second trimester, birthweight Z-Score, cord blood insulin, gestational age at delivery, and neonatal sex. GWG was adjusted for gestational age at weight measurement in mid-pregnancy. Cord blood insulin was adjusted for sample storage duration. All continuous variables were standardized to Z-Scores to eliminate the influence of different measurement units. Mother-child pairs with missing data were excluded(n=93/1522, 6\%).

Firstly, an optimal DAG with the best goodness of fit(highest log marginal likelihood) was identified. Next, parametric bootstrapping(12800 samples) was performed to address the potential overfitting. Full technical details are provided in the supplementary file(S5). ABN analysis was conducted in R 3.4.4(The R Foundation for Statistical Computing) using 'abn' package(11).

\section{Results}

Figure 1 shows the flow diagram of mother-child pairs' selection procedure(power calculation provided in supplementary file S1). The baseline characteristics of participants are shown inTable 1 . The majori$\operatorname{ty}(91.20 \%)$ of maternal blood samples were collected after overnight fasting. Maternal mid-pregnancy weight, fasting glucose, and lipids profile were measured at a mean of $20.0(\mathrm{SD}=4.0), 24.6(\mathrm{SD}=1.4)$, and $20.5(\mathrm{SD}=3.5)$ gestation weeks, respectively. Cord blood samples were stored for a median of 488(IQR 394 to 707) days before analysis.

\section{Association of maternal metabolic parameters with birthweight and CBI}

Table 2 presents the associations between the association of maternal pre-pregnancy BMI, GWG, and maternal mid-pregnancy fasting glucose/HDL-C/TG levels with neonatal outcomes. Pre-pregnancy $\mathrm{BMI}\left(\right.$ adjusted $\beta=29.25,95 \% \mathrm{CI}: 22.77$ to $35.73 \mathrm{~g}$ per $\left.\mathrm{Kg} / \mathrm{m}^{2}\right), \mathrm{GWG}$ (adjusted $\beta=18.75,95 \% \mathrm{CI}$ : 13.06 to $24.43 \mathrm{~g}$ per $\mathrm{Kg}$ ), fasting glucose (adjusted $\beta=84.32$, $95 \% \mathrm{CI}: 42.65$ to $125.98 \mathrm{~g}$ per $\mathrm{mmol} / \mathrm{L}$ ), and triglycerides(adjusted $\beta=67.97,95 \%$ CI: 42.38 to $93.55 \mathrm{~g}$ per $\mathrm{mmol} / \mathrm{L}$ ) were positively associated with birthweight, while HDL-C was negatively associated with birthweight(adjusted $\beta=45.78,95 \%$ CI: 5.59 to $85.97 \mathrm{~g}$ per 
mmol/L). There was no evidence of an association between maternal TC and LDL-C levels and birthweight(Supplementary file S6).

Elevated maternal pre-pregnancy BMI, early GWG, fasting blood glucose and triglycerides level were significantly associated with an increased risk of LGA. The risk was particularly high for fasting glucose $(\mathrm{OR}=2.06$, 95\% CI 1.31 to 3.24). Higher pre-pregnancy BMI and triglycerides were significantly associated with lower odds of SGA. There was no evidence of an association between maternal HDL-C level and risk of LGA/SGA.

Maternal fasting glucose(adjusted $\beta=2.23,95 \% \mathrm{CI}$ : 0.89 to $3.57 \mu \mathrm{U} / \mathrm{ml}$ per $\mathrm{mmol} / \mathrm{L}$ ) and triglycerides levels(adjusted $\beta=0.88,95 \%$ CI: 0.05 to $1.71 \mu \mathrm{U} / \mathrm{ml}$ per $\mathrm{mmol} / \mathrm{L}$ ) were significantly associated with higher CBI. Pre-pregnancy BMI, GWG, TC, and LDL-C levels did not show an association with CBI(Table 2 and Supplementary file S6).

\section{Association of maternal metabolic parameter Z-scores with birthweight and CBMI Z-scores}

Table 3 shows the estimates of the association of maternal metabolic parameter Z-Scores with birthweight Z-Score and CBI Z-Score, and subgroup estimates for boys and girls. Maternal pre-pregnancy $\mathrm{BMI}$ (adjusted $\beta=0.20,95 \% \mathrm{CI} 0.15$ to 0.24 ), early $\mathrm{GWG}(\operatorname{adjusted} \beta=0.17,95 \% \mathrm{CI} 0.12$ to 0.22 ), triglycerides(adjusted $\beta=0.12,95 \%$ CI 0.08 to 0.16 ), and glucose(adjusted $\beta=0.08,95 \%$ CI 0.04 to 0.12 ) Z-scores had positive associations with birthweight Z-Score. Maternal HDL-C Z-Score showed a negative association with birthweight Z-Score in boys(adjusted $\beta=-0.06,95 \% \mathrm{CI}-0.12$ to -0.01 ) only. The association of pre-pregnancy BMI, GWG, glucose, and triglycerides Z-Scores with birthweight Z-Score remained statistically significant after adjusting for other four maternal metabolic risk factors.

Maternal glucose Z-Score(adjusted $\beta=0.13,95 \%$ CI 0.08 to 0.18 ) appears to be the most important contributor to CBI Z-Score in both boys and girls. Both maternal pre-pregnancy BMI(adjusted $\beta=0.10,95 \% \mathrm{CI} 0.05$ to 0.15 ) and triglycerides(adjusted $\beta=0.06,95 \%$ CI 0.01 to 0.11 ) Z-Scores showed positive associations with CBI Z-Score. No statistically significant association was observed between maternal early GWG and HDL-C Z-Scores with CBI Z-Score.

\section{ABN analysis results for interdependent maternal metabolic parameters}

Figure 2 shows the optimal summary DAGs inferred by ABN analysis(Supplementary file S5). The adjusted regression coefficients $(\beta)$ in the graph represent how much the dependent variable changes per unit increase in the independent variable. Maternal pre-pregnancy BMI appeared to be the most influential upstream factor for both maternal metabolic parameters in pregnancy(glycaemia: $\beta=0.14,95 \% \mathrm{CI} 0.09$ to 0.19 ; early GWG: $\beta=-0.12,95 \%$ CI -0.17 to -0.06 ; triglycerides: $\beta=0.23$, 95\% CI 0.18 to 0.28 ; HDL-C: $\beta=-0.12,95 \%$ CI -0.17 to $-0.07)$ and birthweight $(\beta=0.27,95 \% \mathrm{CI} 0.22$ to 0.32$)$. An indirect effect on neonatal insulin secretion was also observed. Our DAGs results showed that maternal glycaemia was associated with $\mathrm{CBI}(\beta=0.12,95 \% \mathrm{CI} 0.07$ to 0.17$)$. Birthweight was also associated with $\operatorname{CBI}(\beta=0.24,95 \% \mathrm{CI} 0.19$ to 0.29$)$. Neither triglycerides nor HDL-C were linked to birthweight or CBI.

\section{Discussion}

To our knowledge, this is perhaps the first large prospective birth cohort study to map the metabolic network, and assess the inter-dependent associations between maternal modifiable metabolic risk factors, birthweight, and insulin secretion in neonates. We showed that high maternal pre-pregnancy BMI appears the most influential upstream metabolic risk factor for both maternal and neonatal health. Maternal early GWG is directly associated with birthweight, but not neonatal insulin secretion. Maternal fasting glucose is significantly associated with increased neonatal insulin secretion. Although maternal HDL-C and triglycerides concentrations are significantly associated with birthweight, our results demonstrated that these lipid pathways may not 
be meaningfully involved in the metabolic network pathway between mothers and neonates, and instead be a proxy measure for maternal metabolic health. These findings suggest: 1) the primary focus of weight management in clinical practice to prevent adverse pregnancy outcomes should start from preconception; 2) the observed association between maternal glucose and birthweight is likely to be partly mediated through elevated neonatal insulin secretion; 3) The pathogenic relationships of maternal glucose/triglycerides with birthweight/CBI need to be inferred with caution and evaluated in further studies.

Our results are generally consistent with previous relevant evidence, but importantly we also provide new insights that differ from the conclusions of previous studies. The Hyperglycaemia and Adverse Pregnancy Outcome Study research group(HAPO) published a series of network analyses reporting that maternal metabolites(acylcarnitines, fatty acids, carbohydrates, and amino acids) during pregnancy are associated with BMI, fasting glucose, and insulin resistance in mothers(18) and birth size, growth, adiposity, and cord blood C-peptide in neonates $(8,19)$. Consistent with our findings, another study that looked at all metabolic parameters but not in the context of network analysis and restricted to women with GDM only $(\mathrm{n}=357)$ reported that the number of altered maternal metabolic characteristics(pre-pregnancy BMI, fasting glycaemia, HbA1c, triglycerides, and HDL-C) are associated with incidence of LGA(9). However, none of those studies explored theinter-dependent relationships between maternal metabolic risk factors and compared the strength of their associations with neonatal conditions.

Maternal high pre-pregnancy BMI has been linked with increased birthweight(20). Beyond that, our analyses demonstrated that maternal pre-pregnancy BMI is the most important contributor to increased birthweight, which is independent of maternal early GWG, glucose, and triglycerides levels during pregnancy. It is also worth noting that high maternal pre-pregnancy BMI is closely related to gestational metabolic disorders, namely, increased fasting glucose and triglycerides levels, therefore, further contributing to elevated birthweight and insulin secretion in offspring. Similar to our results, a small study containing 66 mother-offspring pairs found that obese mothers might induce increased insulin secretion in offspring. Their results also showed a clear sexual dimorphism(boys have higher insulin secretion than girls)(21). In this study, the association between maternal pre-pregnancy BMI with CBI in boys seemed stronger than in girls $(\beta[95 \% \mathrm{CI}]$, boys $0.13[0.07,0.20]$ vs. girls $0.08[0.00,0.15])$, but the difference was not statistically significant. On the other hand, we found that maternal early GWG is only statistically associated with birthweight, but not CBI, which suggests that the weight accumulation in the early pregnancy may indirectly affect neonatal metabolism through increased birthweight.

Maternal glucose has been closely associated with increased birthweight and cord blood C-peptide levels(1, 22 ). Our results of multivariable regression model are in line with previous findings. The ABN results suggest that maternal fasting glucose is perhaps not directly linked with birthweight, while the concentration of cord blood insulin is largely determined by birthweight and maternal glucose jointly. When we entered CBI Z-Score in the regression model, the association between maternal glucose Z-Score and birthweight Z-Score decreased dramatically but remained statistically significant $(\beta=0.05,95 \%$ CI 0.01 to 0.09$)$. This suggests that maternal hyperglycaemia drives neonatal insulin secretion, and the de novo anabolic effect of CBI plays a critical role in adipose accumulation in neonates $(23,24)$. The enlarged adipocyte will gradually become resistant to insulin to avoid further expansion, therefore contributes to the increased insulin secretion in neonates $(25)$.

We recently published a systematic review which found that increased maternal triglycerides and decreased HDL-C are positively associated with high birthweight(26). Similar results were observed using multivariate regression analysis in this study. However, our ABN analysis now take us a step further by suggesting that both maternal HDL-C and triglycerides are likely to be measures of gestational metabolic disorder, and not themselves involved in the metabolic pathway that increases birthweight and CBI. Similar to our results, a Mendelian randomization study analysing data from 30,487 women in 18 studies concluded that genetically higher maternal fasting HDL-C/triglycerides was not potentially causally associated with higher birthweight(27). Thus, both detailed pathways analyses in this paper and genetic findings go against lipid pathways being directly relevant to birthweight. 


\section{Clinical Implication}

Most current clinical guidelines on preconception and antenatal care only focus on weight management during pregnancy. Our results provide further important evidence on the clinical importance of maternal pre-pregnancy high BMI for both maternal and neonatal health outcomes. Interventions to reduce weight in overweight/obese women before conception to reduce adverse effects of high maternal pre-pregnancy BMI may need further investigation in randomized trials. Recommendations on pre-pregnancy weight management is limited and ambiguous(28-30). None of guidelines on weight management in adults provides advice to women in child-bearing age to prevent adverse pregnancy outcomes. Only one public health guideline in UK mentions the potential course of actions that could be taken by health professionals to improve outcomes in women with a BMI equal or in excess of $30 \mathrm{~kg} / \mathrm{m}^{2}$ prior to pregnancy(28). Our results, if applied to wider communities, provide further evidence for public health measures at improving weight levels in women in general and particularly those of child-bearing age.

\section{Strengths and limitations}

The major strengths of this study are the prospective design based on relatively large sample size, standardization of strength of association for the comparison among maternal metabolic risk factors, and the use of powerful analytical tools for interpretation of multi-dimensional data. Given the practical constraints, maternal fasting glucose and triglycerides levels were measured only once during pregnancy. Therefore, we could not investigate the dynamic long-term influences of maternal metabolic risk factors in detail, although such levels generally track well over gestation. The average pre-pregnancy BMI of included women and incidence of LGA/SGA babies in this study were significantly lower than for people living in the northern part of China. The relative healthiness of our cohort suggests that our results might underestimate the true impact of maternal metabolic disorders on neonatal health outcomes if extrapolated to this wider population. The pre-pregnancy weight was self-reported, which might potentially underestimate the true value. However, evidence suggests that utilization of self-reported or measured pre-pregnancy weight for pre-pregnancy BMI classification results in identical categorization for most women(31). In addition, due to lacking of dynamic data, the ABN analysis might have limited ability on exploring feedback loop. Therefore, the results of $\mathrm{ABN}$, as with any observational analyses, need to be interpreted with a degree of caution.

\section{Conclusion}

In conclusion, in this cohort study, high maternal pre-pregnancy BMI appeared to be the most influential upstream risk factor for gestational hyperglycaemia/hypertriglyceridemia in mothers and increased birthweight/insulin secretion in neonates. Our findings based on robust novel statistical methods highlights the impact of maternal pre-pregnancy BMI on maternal and neonatal metabolic outcomes. We also found that maternal hyperglycaemia was positively associated with elevated neonatal insulin secretion, suggesting this may be a key reason for foetal fat deposition. By contrast, maternal lipid levels do not appear to have meaningful independent associations with neonatal birthweight or CBI.

\section{Grants and/or financial support:}

This work was supported by National Natural Science Foundation of China [grant numbers 81673181, 81703244, and 81803251]. JW was supported by the LiSiguang scholarship provided by the University of Birmingham and the China Scholarship Council jointly. The funder had no role in study design, data collection, data analysis, data interpretation, or writing of the report. The corresponding author had full access to all the data in the study and had final responsibility for the decision to submit for publication.

Disclosure Statement : The authors report no conflict of interest.

\section{Contribution statement:}


$\mathrm{KN}, \mathrm{QX}, \mathrm{KKC}$, and JW conceived the research question. JW defined the question, designed the study, and conducted data analysis. KN and MJP advised on study design and contributed to data analysis. KKC, HX, and QX provided input for study design. YK, JH, SS, JL, and JW contributed to the sample collection, storage, and biochemical tests, and data pre-processing. MP checked the ABN analysis externally. All authors contributed to the interpretation of the results. JW led the writing of the manuscript with critical input from all other authors. JW, XQ, and KN had full access to all data (including statistical reports and tables) in the study and can take responsibility for the integrity of the data and the accuracy of the data analysis. JW is the guarantor.

Data sharing: No additional data available.

Transparency: The lead author (JW) affirms that this manuscript is an honest, accurate, and transparent account of the study being reported; that no important aspects of the study have been omitted; and that any discrepancies from the study as planned (and, if relevant, registered) have been explained.

Ethical approval: Consent has been obtained from each patient or subject after full explanation of the purpose and nature of all procedures used.

\section{References}

1. HAPO Study Cooperative Research Group. Hyperglycemia and adverse pregnancy outcomes. New England Journal of Medicine. 2008;358(19):1991-2002.

2. Villamor E, Cnattingius S. Interpregnancy weight change and risk of adverse pregnancy outcomes: a population-based study. The Lancet. 2006;368(9542):1164-70.

3. American Diabetes Association. 2. Classification and diagnosis of diabetes. Diabetes care. 2015;38(Supplement 1):S8-S16.

4. Ng M, Fleming T, Robinson M, Thomson B, Graetz N, Margono C, et al. Global, regional, and national prevalence of overweight and obesity in children and adults during 1980-2013: a systematic analysis for the Global Burden of Disease Study 2013. The lancet. 2014;384(9945):766-81.

5. Rogozińska E, Marlin N, Jackson L, Bogaerts A, Rayanagoudar G, Ruifrok AE, et al. Effects of antenatal diet and physical activity on maternal and fetal outcomes: individual patient data meta-analysis and health economic evaluation. Health Technol Assess. 2017;21(41):1-+.

6. Barrett HL, Nitert MD, McIntyre HD, Callaway LK. Normalizing metabolism in diabetic pregnancy: is it time to target lipids? Diabetes care. 2014;37(5):1484-93.

7. Heerwagen MJ, Miller MR, Barbour LA, Friedman JE. Maternal obesity and fetal metabolic programming: a fertile epigenetic soil. Am J Physiol Regul Integr Comp Physiol. 2010;299(3):R711-22.

8. Scholtens DM, Bain JR, Reisetter AC, Muehlbauer MJ, Nodzenski M, Stevens RD, et al. Metabolic networks and metabolites underlie associations between maternal glucose during pregnancy and newborn size at birth. Diabetes. 2016:db151748.

9. Zawiejska A, Wender-Ozegowska E, Brazert J, Sodowski K. Components of metabolic syndrome and their impact on fetal growth in women with gestational diabetes mellitus. J Physiol Pharmacol. 2008;59(Suppl 4):5-18.

10. Schaefer-Graf UM, Graf K, Kulbacka I, Kjos SL, Dudenhausen J, Vetter K, et al. Maternal lipids as strong determinants of fetal environment and growth in pregnancies with gestational diabetes mellitus. Diabetes Care. 2008;31(9):1858-63.

11. Pittavino M, Lewis F, Furrer R. abn: an R package for modelling multivariate data using additive Bayesian networks. The Comprehensive R Archive Network (CRAN), 1-37. 2016. 
12. Koivisto M, Sood K. Exact Bayesian structure discovery in Bayesian networks. Journal of Machine Learning Research. 2004;5(May):549-73.

13. Qiu X, Lu J-H, He J-R, Lam K-bH, Shen S-Y, Guo Y, et al. The Born in Guangzhou Cohort Study (BIGCS). European Journal of Epidemiology. 2017;32(4):337-46.

14. International Association of Diabetes: Pregnancy Study Groups Consensus Panel. International association of diabetes and pregnancy study groups recommendations on the diagnosis and classification of hyperglycemia in pregnancy. Diabetes care. 2010;33(3):676-82.

15. Chen $\mathrm{C}, \mathrm{Lu} \mathrm{F}$. The guidelines for prevention and control of overweight and obesity in Chinese adults. Biomedical and environmental sciences: BES. 2004;17:1-36.

16. Villar J, Papageorghiou AT, Pang R, Ohuma EO, Ismail LC, Barros FC, et al. The likeness of fetal growth and newborn size across non-isolated populations in the INTERGROWTH-21 st Project: the Fetal Growth Longitudinal Study and Newborn Cross-Sectional Study. Lancet Diabetes Endocrinol. 2014;2(10):781-92.

17. Edison RJ, Berg K, Remaley A, Kelley R, Rotimi C, Stevenson RE, et al. Adverse birth outcome among mothers with low serum cholesterol. Pediatrics. 2007;120(4):723-33.

18. Sandler V, Reisetter AC, Bain JR, Muehlbauer MJ, Nodzenski M, Stevens RD, et al. Associations of maternal BMI and insulin resistance with the maternal metabolome and newborn outcomes. Diabetologia. 2017;60(3):518-30. doi: 10.1007/s00125-016-4182-2. Epub 2016 Dec 16.

19. Kadakia R, Nodzenski M, Talbot O, Kuang A, Bain JR, Muehlbauer MJ, et al. Maternal metabolites during pregnancy are associated with newborn outcomes and hyperinsulinaemia across ancestries. Diabetologia. 2018;27(10):018-4781.

20. Zhu Y, Zhang C. Prevalence of gestational diabetes and risk of progression to type 2 diabetes: a global perspective. Current diabetes reports. 2016;16(1):7.

21. Mingrone G, Manco M, Mora MEV, Guidone C, Iaconelli A, Gniuli D, et al. Influence of maternal obesity on insulin sensitivity and secretion in offspring. Diabetes care. 2008;31(9):1872-6.

22. He XJ, Qin FY, Hu CL, Zhu M, Tian CQ, Li L. Is gestational diabetes mellitus an independent risk factor for macrosomia: a meta-analysis? Archives of Gynecology and Obstetrics. 2015;291(4):729-35.

23. Petersen MC, Shulman GI. Mechanisms of insulin action and insulin resistance. Physiological reviews. 2018;98(4):2133-223.

24. Lawlor DA, West J, Fairley L, Nelson SM, Bhopal RS, Tuffnell D, et al. Pregnancy glycaemia and cord-blood levels of insulin and leptin in Pakistani and white British mother-offspring pairs: findings from a prospective pregnancy cohort. Diabetologia. 2014;57(12):2492-500.

25. Varlamov O, Somwar R, Cornea A, Kievit P, Grove KL, Roberts Jr CTJ. Single-cell analysis of insulinregulated fatty acid uptake in adipocytes. American Journal of Physiology-Endocrinology \& Metabolism. 2010;299(3):E486-E96.

26. Wang J, Moore D, Subramanian A, Cheng K, Toulis K, Qiu X, et al. Gestational dyslipidaemia and adverse birthweight outcomes: a systematic review and meta-analysis. Obes Rev. 2018.

27. Tyrrell J, Richmond RC, Palmer TM, Feenstra B, Rangarajan J, Metrustry S, et al. Genetic evidence for causal relationships between maternal obesity-related traits and birth weight. Jama. 2016;315(11):1129-40.

28. NICE. Weight management before, during and after pregnancy. 2010.

29. Rasmussen KM, Yaktine AL. Weight gain during pregnancy: reexamining the guidelines: National Academies Press; 2010. 
30. Royal College of Obstetricians and Gynaecologists. Care of women with obesity in pregnancy (Green-top Guideline No. 72). 2018 Nov 22.

31. Bannon AL, Waring ME, Leung K, Masiero JV, Stone JM, Scannell EC, et al. Comparison of selfreported and measured pre-pregnancy weight: implications for gestational weight gain counseling. Maternal and child health journal. 2017;21(7):1469-78.

\section{Hosted file}

Figure 1 Flow chart.docx available at https://authorea.com/users/297717/articles/426838maternal-metabolic-risk-factors-and-their-association-with-birthweight-and-cord-bloodinsulin

\section{Hosted file}

Figure 2 Additive Bayesian Network Graph.docx available at https://authorea.com/users/297717/ articles/426838-maternal-metabolic-risk-factors-and-their-association-with-birthweightand-cord-blood-insulin 